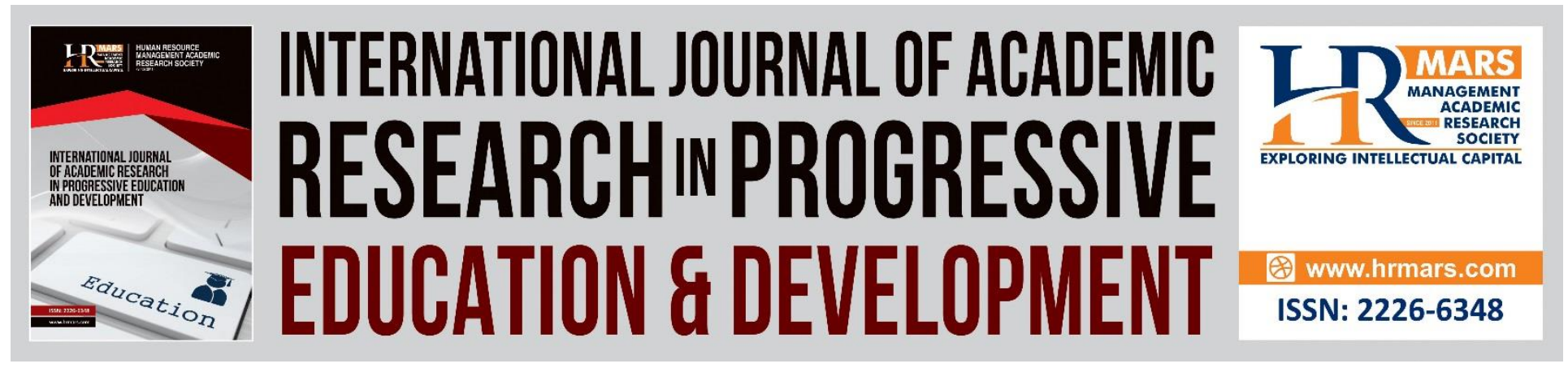

\title{
Implementation of Electronic Medical Records in Developing Countries: Challenges \& Barriers
}

Nik Azliza bt Nik Ariffin, Aliza bt Ismail, Irwan Kamaruddin Abdul Kadir and Jannatul Iza Ahmad Kamal

To Link this Article: http://dx.doi.org/10.6007/IJARPED/v7-i3/4358

DOI: $10.6007 /$ IJARPED/v7-i3/4358

Received: 09 June 2018, Revised: 23 June 2018, Accepted: 03 July 2018

Published Online: 23 July 2018

In-Text Citation: (Ariffin, Ismail, Kadir, \& Kamal, 2018)

To Cite this Article: Ariffin, N. A. bt N., Ismail, A. bt, Kadir, I. K. A., \& Kamal, J. I. A. (2018). Implementation of Electronic Medical Records in Developing Countries: Challenges \& Barriers. International Journal of Academic Research in Progressive Education and Development, 7(3), 187-199.

Copyright: (c) 2018 The Author(s)

Published by Human Resource Management Academic Research Society (www.hrmars.com)

This article is published under the Creative Commons Attribution (CC BY 4.0) license. Anyone may reproduce, distribute, translate and create derivative works of this article (for both commercial and non-commercial purposes), subject to full attribution to the original publication and authors. The full terms of this license may be seen at: http://creativecommons.org/licences/by/4.0/legalcode

\section{Vol. 7, No. 3, July 2018, Pg. 187 - 199}




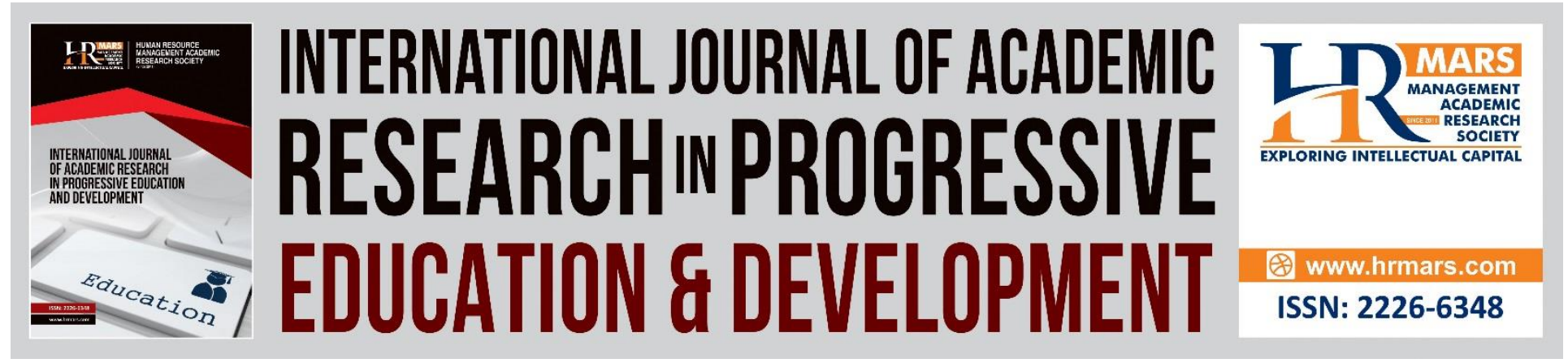

\title{
Implementation of Electronic Medical Records in Developing Countries: Challenges \& Barriers
}

\author{
Nik Azliza bt Nik Ariffin, Aliza bt Ismail, Irwan Kamaruddin Abdul \\ Kadir and Jannatul Iza Ahmad Kamal \\ Faculty of Information Management, Universiti Teknologi MARA, UiTM Selangor, Malaysia
}

\begin{abstract}
The advancement of technology has changed the way the entire of healthcare industry functions. An electronic medical record is one of the advancement of technology that is significantly and gives a great benefit in medical field. The development of electronic medical record can help to increase the productivity and profit of medical institutions. Most countries in developed countries are increasingly using an electronic medical record (EMR) to improve work process, patient safety and quality of healthcare. Unfortunately, most developing countries address many drawbacks and barriers in implementing EMR. The primary thrust of this paper is to examine the challenges and benefits of an EMR and its contribution to the development of healthcare delivery in developing countries. Some benefits of an EMR system to promote better health care by improving all aspects of patient care and encouraging healthy lifestyles among communities, increase efficiencies and lower health care costs and enhancing in clinical decision making. Despite of EMR challenges facing the developing world such as interoperability, privacy and confidentiality, organizational and social barriers, limitations of technology, electronic preservation, legal status of EMR and customization. The potential of EMRs will make significantly augmentation to health care transformation. Most studies shown how workable it could be with support and enlighten from developed nations to design and implement an EMR system that fits into this setting.
\end{abstract}

Keywords: Electronic Medical Record, Healthcare, Developing Countries, Information Management

\section{Introduction}

"Paper records are increasingly becoming obsolete and inadequate. They limit the flow of information, insufficiently document patient care, impede the integration of health care delivery, create barriers to research, and limit the information available for administration and decision making." (Roy Romanow, 2002) 
Vol. 7, No. 3, July 2018, E-ISSN: 2226-6348 @ 2018 HRMARS

The awareness of health care quality has been growing for many years and this has been significant issue to the Ministry of Health $(\mathrm{MOH})$ Malaysia. One characteristic that needs to be considered in improving quality of health care is records management. With the shift in technology, implementation of electronic medical records (EMR) is arising and thus, evolving the health care environment.

The phrase EMR itself has underwent changes which set out from "computer stored medical records" subsequently computerized patient record (CPR), computerized medical record (CMR), computer-based patient record system (CBPR), electronic health record (EHR), and automated medical record (AMR) (Fisher,1999).

Under The Seventh Malaysian Plan (Government of Malaysia, 1996) EMR system implementations in Malaysia started out in the year 1999. Since then the system commenced in phases throughout the country. At present, 21 out of 138 public hospitals which made up 15.2\% public hospitals implemented the system (Ismail et al, 2015).

The use of information technology (IT) implemented in EMR is now widely accepted in hospitals as well as clinics. This technology based improvement, is seen to make better exchange of information and bring out more effective communication among doctors. Implementation of EMR is also seen as a promising IT based solution in health care quality improvement (Gastaldi et al, 2012). This is due to the competency of EMR system to handle large amount of information and data within the health system and hence capable of supporting the diverse needs of clinical, organizational and management of health care (Reina et al, 2012).

The EMR system integrates clinical data, patients' records, decision support application programmes and transaction processes within a hospital. With these integration, the system has a potential big impact on the hospital performance (Gastaldi et al, 2012). From a doctor's point of view, EMR allows a better way to collect, store, retrieve and analyze medical information. The analyzed information can then be used to seek the best treatment for a patient and in turn provide a quality care for the patient (Richards, et al 2012). With schematic connections and interdisciplinary understanding, EMR implementation will be able to provide effective and efficient patient, physician and clinic management as well as enhancing good outcome.

\section{Traditional Paper Based Record Keeping.}

Historically, paper based of medical records have been used for centuries. By using paper records have many advantages of being simplicity, low implementation cost, wide spread acceptance, mobile and can entry of subjectivity data. However, yet paper records have many limitations; as paper records is a very fragile medium that requires a big space purposely for storage and it must be properly organized and can be accessible, data recorded may not in a uniformity standard, only one person can access at a time, papers may be missing or lost, misplaced or it lead to complexity and unmanageable, frequent illegibility in clinical notes, lack of capacity to be accessed remotely or cannot be accessed across different locations(Sridhar GR et al,2009), growing so thick more than hundred pages and involving with different volumes and various storage sites. The most significant problem dealing with paper records is about timing process 
Vol. 7, No. 3, July 2018, E-ISSN: 2226-6348 @ 2018 HRMARS

and usually medical professionals need the patient data instantly and time is very critical in healthcare organizations. Paper is not a stable media format, and by nature easily affected to both water and fire. All medical records need to be locked in a storage area in order to protect confidentiality, integrity, and security of the information.

\section{Transition from Paper-Based to EMR systems}

Conversion all the information in paper medical records to electronic medical records provides significant clinical and operational benefits

A successful transition from paper-based to electronic medical records (EMRs) in the healthcare organizations require careful supervision and coordination of many aspects. A manifold of drawbacks and big decisions must be made and it begins from selection and implementation, training and maintenance. The transition involves not only the process changes but also the using of a new tool technically and procedural training and lead changes to roles of healthcare professionals within the office. (College of Physicians and Surgeons of Alberta Guideline, 2005)

EMR adoption will not success if they fail to evaluate the clinical workflows and information needs and lack of planning during and after go-live and the result of this will fall back to paper. To make it successful, the practice must fully understand the expected advantages and must analyze each of the choices to ensure the whole practice workflow is considered (Ronald A. et al, 2010)

\section{Electronic Medical Records}

According to Thielst (2007a) information technology adoption in hospitals provides safer, more effective and efficient in healthcare delivery systems that transforms healthcare. Technology may be effectively used in health care industry to improve procedures, common practice, regulations, public standards and protocols to produce great patient outcomes and enhanced patient safety. IT becoming a key driver of effective business and clinical enterprises.

The advancement of electronic medical record (EMR) technology have made it possible for the EMR to replace many functions of the traditional paper chart, and use of EMR systems promises significant advances in patient care.(Institute of Medicine,1997)

An electronic medical record (EMR) exists in electronic version of the paper-based record. It is a computer-based system for storing, organizing, and retrieving information about patients, holds tremendous expectation for improving the quality and safety of healthcare. An electronic medical record (EMR) can embrace much information such as name in full, complete address, date of birth, gender, person to notify in an emergency, insurance, medications details of present and past history, allergies, laboratory and test results; immunization, medical surgical, and hospitalization history; as well as the documentation of the patient's progress assessment, vital signs, plan of care, education and research that can be accessed from various sites within the hospital under the protection of security, patient privacy and confidentiality. 
Vol. 7, No. 3, July 2018, E-ISSN: 2226-6348 @ 2018 HRMARS

The term of EMR defines as a computerized medical records that can be accessed from various integrated system at any point of care within the health care enterprise with concerned of patient privacy, confidential and security. (Wagner, 2004).

Electronic medical records (EMRs) exist in electronic format instead of paper (Sridhar GR et al,2009).The situation before using this new technology was huge different from the effects after its implementation. The situation before EMRs implementation, manually patient record keeping in paper records format. As much of the information in the paper based records was hand written and usually difficult to read, transcribe, and understand as it written by healthcare professionals then it leads to medical errors. "High failure rates towards paper-based systems for information storage and retrieval that can lead to duplication of services, delays in treatment, increased duration of stay in hospital and increased errors in medical due to absence or inaccessibility of data" (Crane \& Raymond, 2003) By having electronic medical record system as a tool to facilitate a centralized patient information repository and to provide documented records of care that supports present and future care by the healthcare professionals and also as medium of communication among healthcare professionals contributing to the patient's care.

\section{Models of Successful EMR Implementation}

Healthcare is moving forward to a new paradigm shift in health information technology. The need to transform the developing countries healthcare system by implementation of electronic medical record system's functions and features leads the healthcare industry into the new era of 21st century.

Australia, England, New Zealand and the Netherlands are countries that showed success in EMR implementation. (Kidd MR \& Mazza D,2000) But the most tremendously success is Australia as results have been most dramatic. In May 2000, majority of clinicians in their practice were using a computer in their consulting room to write the prescriptions, compared with only $15 \%$ of general practitioners reporting computer use for any purposes in October 1997.(Kidd MR \& Mazza D,2000)

Australia achieved this noteworthy transition by providing general practitioners with financial support to help them purchase a computer, supporting system implementation for those who needed it, and offering incentives for those using electronically in their practices. England has made greater development, albeit more slowly. Currently, 98\% of general practitioners have access to an EMR on their desktop. Nearly all use it purposely for prescription refills, and $30 \%$ report that their practices are paperless.

\section{Advantages Of EMR In Developing Countries}

Electronic medical records are the surge of the future. EMRs represent an attempt to translate information from paper records into a computerized format. Electronic medical records are expected to provide a wide range of advantages to healthcare industry (Fraser HSF et al, 2005). EMRs offer many potential advantages over traditional paper-based records and it is the first step to transformed health care. This electronic medical record is very readable, thus eliminating errors often made when transcribing hand-written information. 
Improving patient care and safety was stated as the main factors driving the need for EMR. Mainly addressed to increase efficiency, accuracy, accessibility, and safety that EMR will bring to the healthcare organizations.

In an ideal situation, the electronic medical record (EMR) is an enabling technology that focus on improvement in quality management. Basically, it concerns on streamline the work processes and accountability, improved results management and patient care with a reduction in errors and legibility of records. According to Lamont (2005), almost $70 \%$ medication problems improved when EMR is implemented.

In clinical productivity, although EMR in a single entry but it provides multiple use for healthcare professionals in hospital. Electronic medical records have the capability to capture and store a huge amount of patient information such a large database results in quality improvement and cost effectiveness (Sidorov, 2006). The data layout is so flexible, automatic in data capturing such as ICU lab and also provide automatic patient chart to increase accuracy and completeness of the record.

EMR contains information only from respective hospital. Yet the ability having this information in a structured way lead to structured data that can enhance and extend research capabilities.

EMR offer the ability to improve patient safety by providing care for patients in a consistent and timely manner in producing results, avoid duplicates and it need to be complied with clinical protocols standards and regulations. Improved efficiencies and lower health care costs by promoting single patient records with integrated information to ensure continuity of care and improved coordination of health care services, as well as by reducing waste and redundant tests

This efficiency can create better clinical decision making by integrating patient information from numerous sources. Sharing patient charts and medical information with other health care providers is also easier by using EMR system as patient centeredness. In paper patient record keeping, still implement data analysis, capture, compilation, consolidation, and reporting but it takes up much time as the process is conducted manually. EMR storage of data permits for improved quality measurement and enhanced clinical research (Halamka, 2006). Once the transition to an EMR system is completed, storage space dedicated to paper charts can be eliminated.

Besides improving the delivery of health care to patients, electronic medical records can decrease administrative process and other related costs for clinicians. EMR implementation can optimize resources as more workload with the same amount of people, can reduce operational costs such as transcription services and overtime labor expenses. Likewise, medical records clerk for filing and retrieval for a patient files and the need to purchase paper based supplies is slowly reduced or eliminated. In terms of revenue enhancements, EMR provides the ability to more accurately and efficiently process patient billing and the exact amount of charge of every patients. Other advantages is the space saving of a digital records environment. 
Financially, from the aspect of micro accounting EMR system can compare performance by unit, care providers and can helps in resource allocation.

Furthermore, virtually all EMR systems improves stakeholders satisfaction by improving access to healthcare information. In administration aspect, EMR improves waiting time especially on appointment basis and scheduling. EMR provide the ability to ultimately increase the number of patients served per day for enhanced patient workflow and increased productivity.

The various points of views about EMRs address both positive and negative remarks. EMR is considered as one of medium communication in healthcare delivery and it can improves transparency and communication among healthcare professionals. EMR provides regulatory compliance to ensure data for statistics and disease notification updated. In legal aspects, EMRs concern on confidentiality, data integrity, access control and audit trail as implementing EMR less prone to data loss.

\section{Challenges and Barriers}

With the promising effects of EMR implementation in developing countries, more health care institutions began to adopt the technology. However, the widespread application of EMR revealed many issues of dispute in the EMR system.

\section{Interoperability}

EMR system integrates all of patients' demographic, clinical and financial data which enables doctors and other health care providers to share the data and make effective decisions in providing a quality care for patients. EMR system also incorporates appointment schedules, billings, and electronic filing as well as coding in order to ensure clinic operations run smoothly.

As demonstrated from above, there is a need of high level integration between the different activities in health care. The complexity of integrating different activities can be seen from the operational treads interdepartmentally. Clinical department involves history taking, giving diagnosis and planning for treatments. Supportive department includes nursing, allied health fields and laboratory. Administrative department on the other hand observes economic, financial and legal process. Each department would require specialized and detailed technical knowledge. However, in giving care to patients, all staff from the relevant departments need to give cooperation and collaborate to give a quality care (Reina et al, 2012).

With many different activities involvement, there is bound for conflicts to arise. However, without integration and interoperable EMR system, doctors, pharmacists, nurses, supporting staff and hospital management staff could not share patients' information, and in turn taking chances in patients' care. The technical interdependencies require coordination of information that links people and actions which in turn shapes the outcome of care process.

In order to gain wider accessibility of patients' record, older paper medical record need to be merged together into patients' EMR. This is important in ensuring patients' safety and creates a more efficient patients' management plan as well as saving cost of health care. 
To achieve this, paper based patients' data need to be converted to a digital form. The digital scanning process converts physical records from paper documents or diagnostic devices to EMR in form of images. In performing this, the original format should be kept without losing any medical or legal information.

However, it is worth to note that the conversion process is expensive and a time consuming work. Care should be taken in ensuring the exact content is captured. To make matter even more complicated is that the sources of physical records varies and made up of several formats, sizes and of different media type, all of which effected the accuracy of conversion.

The scanned physical records undeniably important in making the EMR complete. Nonetheless, scanned records are not always usable depending the quality of the image produced and not all doctors are satisfied using images of scanned documents (Lærum et al, 2003). Apart from conversion of records, it is crucial that the original physical records is destroyed in a manner that the records are confidentially and totally destroyed.

\section{Privacy and Confidentiality}

Privacy poses a paramount importance and has been a big challenge in EMR implementation. The ethical dilemma it lays out not only affects patients in the EMR system but also health care providers themselves.

In practice, doctors and all personnel that are involved in health care of a patient are required to protect the patient's information. To ensure this, only authorized personnel can have access to the patient's information. Even so, multiple access points in an open network of an EMR system add on the possibility of patient's information interception.

In an open network, a completely unrelated doctor for example, can locate, request and obtain the medical information of patients. Thus, in this situation, there is a need of authentication of information sharing requests in order to maintain data security (Stanberry, 2011). This step however, delays decision making as a doctor or other personnel might need to wait in order to gain permission to access to a patient's information should they require it (Currie \& Finnegan, 2011). Nonetheless it is crucial in maintaining patient's confidentiality.

\section{Social and Organizational Barriers}

In principle, EMR implementation adopts Pareto's ' $80 / 20$ ' rule where $80 \%$ of work implementation deals with health care and management, and remaining $20 \%$ covers technical issues of the technology itself. Neglecting this aspect would likely lead to barriers in implementing effective EMR system. Such barriers can be in the form of social and organizational barrier.

Social issues are subjected to issues that are effected health care providers in applying EMR technology in their practices. For instance, doctors' attitude towards adopting EMR technology. With increasing widespread utilization of EMR system, doctors experienced a pressing need to document and put in every part of available information in the system (Richards et al, 2012). As a result of this, productivity decreases since this slows down the process of seeing and reviewing patients as doctors are more focused on the computer. This in turn brings a negative perception to patients as they feel they are not getting enough attention and care (Boswell, 2013). 
Vol. 7, No. 3, July 2018, E-ISSN: 2226-6348 @ 2018 HRMARS

On the other hand, doctors of older generation have more trouble in adopting this technology as compared to the younger generation (Cresswell et al, 2011) as they dread uncertainties of the technology and fear of inappropriate technology interface (Boswell, 2013). This lead to a tendency to focus more on clinical aspects as compared to the administrative aspects (Gastaldi et al, 2012).

Organizational issues in turn are issues that involve work flow structure and the challenges to create a positive environments that nurture good communication between health care providers and information technology project managers. Furthermore, EMR implementation needs training programmes and a lot of practices. This is essential especially in transition phase of moving from paper-based system to the electronic system (Richards et al., 2012). The implementation of this technology requires improved performance and constant feedback. If not, the implementation a health care institution will experience more problem as compared to the days before the implementation of EMR technology.

\section{Technology Limitations}

Adoption of EMR technology requires administrative technological capabilities. It is important that health care IT administrators infer to the context in which health care providers work in and understand users to gain effective implementation (Richards et al., 2012). It has been proposed that EMR system will be successfully implemented if the EMR software's chosen support and enable use of existing technology. Even so, EMR software systems varies in different hospitals and not standardize throughout the country.

From this, the next logical step in EMR system is digital networking where information is sharable and can be used as a valuable resource to enhance patients' care. This is also a challenge in making patients' information to be mobile as in the current system, patient's data just remained within the facilities where care or treatment has been delivered (Stanberry, 2011). Apart from that, not only the access and use of information that should be looked into, but the quality of information shared is also important in the digital networking.

Another technological drawback of EMR implementation is during the system being down and health care providers of an institution have to go back to paper based system (Boswell, 2013). This might take some times till the system is fully operational again. During this period of time it is mostly feared that a health care provider might possibly miss an important thing that effects patients' care as most of the data is in the EMR system. It is due to this reason that many hospital did not adopt a fully paperless system.

These technological inefficiencies can tie up doctors and other health care providers. In turn it will affect patients' quality of care even those appear to be minor (Shaw et al., 2011). Thus, in order to ensure the effectiveness of EMR system implementation, IT administrators and doctors should review the technology on a regular basis, acquire information on emerging technologies and gain feedback from other doctors as well (Richards et al.,2012). By analyzing this new technologies, EMR system implementation can be improved.

\section{Preserving Electronic Records}

In the EMR system, information and events are preserved and the data will be kept legitimate (Riesenmy, 2010). For example, computerized patient records, patients' care monitoring, information flow and financial data. Apart from that, it will also include physical medical records 
Vol. 7, No. 3, July 2018, E-ISSN: 2226-6348 @ 2018 HRMARS

in the form of films and tracings from diagnostic imaging procedures such as x-ray, CT, PET, MRI, ultrasound, and so forth which is stored in image format. Physical medical records conversion however are time consuming and costly process and must be planned and budgeted.

Even though EMR system has been widely adopted, there is still a fair amount of paper or physical medical records generated. This would require additional space for storage and needs to be handled with as well as kept safe until it can be destroyed (Lott, 1997). On the other hand, digitized medical records are stored in alternative media such as compact disc and tape and thus, the storage would not take up much space. This in turn would seems like that there is no need of destroying the data and retention will be permanent even though the law permits it. As a consequence, the information itself would no longer serve a useful purpose.

\section{Legal status of EMR}

Medical records, be it physically or in the electronic form are legal documents which are the property of the medical provider where it was produced. However, the information in the medical record are owned by the patient and they have the right to view the record and obtain copies the under law. Thus, it is important that the records are kept unaltered and authenticated by those who created the record.

The electronic record poses many medico-legal issues in terms of patients' data transmission and storage. These include protection of patient confidentiality, fraudulence, access restriction and documentation (Stanberry, 2011). Many nation faces these challenges in adopting the EMR system and have enacted their law regarding telemedicine and the use of electronic information. Malaysia however, does not have such law enacted.

\section{Customization}

Most EMR system are based on a model that are general in scope to begin with. These can then be altered accordingly in cooperation with the vendor/developer to better fit a medical specialty, environment or other specified needs as every health care provider has different requirements and needs to be custom tailored. This in turn will affect the pricing of the EMR system adopted.

The cooperation between vendor/developer and health care providers will shape the final product, resulting in the design and impact on the user. (Cresswell et al., 2011). A lack of communication may cause developers to make assumptions on what users needed and wanted. This is further complicated by a lack of perception of a clinical practice by the developers. As a result, health care providers perceived it as lack of fitness for application.

\section{Conclusion}

Information can serve both as an advantage and as a challenge to a health care providers in health care practices nowadays. Introduction of information technology into health care services has provide assistance to health care providers in caring and treating patients.

Adoption of EMR system has shown to reduce weaknesses of paper-based medical records and improve patients' safety. However, there are still many issues revolve around adoption of EMR system and should be looked at closely to further enhance the benefit of EMR. Future research is requires to look at ways to resolve these issues. EMR offer the ability to improve 
INTERNATIONAL JOURNAL OF ACADEMIC RESEARCH IN PROGRESSIVE EDUCATION AND DEVELOPMENT

Vol. 7, No. 3, July 2018, E-ISSN: 2226-6348 @ 2018 HRMARS

patient safety, involve patients in their own care and decrease the overall cost of health care in developing countries.

This healthcare transformation includes technological changes to keep up with the everchanging environment. The adoption of EMR is a step toward healthcare transformation in the developing countries.

\section{References}

Boswell R.A. (2013),"Implementing electronic health records: implications for HR professionals", Strategic HR Review, 12(5): 262 - 268.

Cresswell K, Worth A \& Sheikh A, (2011),"Implementing and adopting electronic health record systems", Clinical Governance: An International Journal, 16 (4): 320 - 336.

Currie W.L. \& Finnegan D.J., (2011),"The policy-practice nexus of electronic health records adoption in the UK NHS", Journal of Enterprise Information Management, 24 (2): 146 - 170.

College Of Physicians And Surgeons of Alberta Guideline. Transition to electronic medical records. (2005), www. cpsa.ab.ca.publicationsresources/attachements_policies/ Transition\%20t0\%20Electronic\%20Medical\%20Records. Pdf

Faustine Williams, M.S. \& Suzanne, A. B. (2008), The role of the electronic medical record (EMR) in care delivery development in developing countries: a systematic review. Informatics in Primary Care, 16:139-45.

Fraser, H.S.F., Biodich, P., Moodley, D., Choi, S., Mamlin, B.W. and Szolovits, P. (2005), Implementing electronic medical record systems in developing countries. Informatics in Primary Care, 83-95.

Fisher J.S, (1999). Electronic Records in Clinical Practice, Clinical Diabetes.

Gastaldi L, Lettieri E, Corso M \& Masella C, (2012) "Performance improvement in hospitals: leveraging on knowledge asset dynamics through the introduction of an electronic medical record", Measuring Business Excellence, 16 (4); 14 - 30

Government of Malaysia (1996). Seventh Malaysian Plan 1996-2000. Kuala Lumpur: National Printers.

Halamka, J. D. (2006). Health information technology: Shall we wait for the evidence? Annals of Internal Medicine, 144(10), 775-776.

Hillestad, R., Bigelow, J., Bower, A., \& Girosi, F. (2005). Can EMR systems transform healthcare? Potential health benefits, savings, and costs. Health Affairs, 24(5), 
Institute of Medicine, (1997), Committee on Improving the Patient Record. The Computer-based Patient Record: An Essential Technology for Health Care. Rev ed. Dick RS, Steen EB, Detmer DE (eds). Washington, DC: National Academy Press.

Ismail, N.I. , Abdullah, N.H. , Shamsuddin, A. (2015) Adoption of Hospital Information System (HIS) in Malaysian Public Hospitals. Procedia - Social and Behavioral Sciences 172: 336 - 343

Kidd, M.R., Mazza, D. (2000) Clinical practice guidelines and the computer on your desk. Med J Austr 2000; 173:373-375.

Lamont, J. (2005). EMRs: A promising prognosis. KM World, 14(8), 12-15.

Lærum H, Karlsen T.H. \& Faxvaag, A. (2003). Effects of Scanning and Eliminating Paper-Based Medical Records on Hospital Physicians' Clinical Work Practice. Journal of the American Medical Informatics Association. 10 (6), 588- 595.

NHS Information Authority, (2002) Building the information Core: Implementing the NHS Plan. Available at: http://www. nhsia.nhs.uk. Accessed August 23.

Lott L.A. , (1997),"The conundrum of patient records", Leadership in Health Services, 10 (2); 4 - 6

Reina R, Cristofaro C, Lacroce A \& Ventura M, (2012),"Managing the knowledge interdependence with electronic medical record", Measuring Business Excellence, (16) 4; 31 - 41

Richards, R.J., Prybutok V.R. \& RyanS, D. (2012),"Electronic medical records: tools for competitive advantage", International Journal of Quality and Service Sciences, 4 (2); 120 - 136.

Riesenmy, K.R. (2010),"Physician sensemaking and readiness for electronic medical records", The Learning Organization, 17(2); 163 - 177

Romanow, R. (2002), Building on values: the future of health care in Canada. Ottawa; 2002.

Shaw, N., Aceti, V., Campbell-Scherer, D., Leyland, M., Mozgala V., Patterson L., Grunfeld, E., (2011),"Current use of electronic medical records in primary care of chronic disease", Clinical Governance: An International Journal, 16(4); 353 - 363

Sidorov, J. (2006). It ain't necessarily so: The electronic health record and the unlikely prospect of reducing health care costs. Health Affairs, 25(4), 1079-1085.

Stanberry, K. (2011),"US and global efforts to expand the use of electronic health records", Records Management Journal, Vol. 21 Iss 3 pp. 214 - 224.

Sridhar, G.R. \& Venkat, Y. (2000) Information technology and endocrine sciences in the 
Vol. 7, No. 3, July 2018, E-ISSN: 2226-6348 @ 2018 HRMARS

new millennium. Indian J Endocrinol Metab 4:70-80.

Sridhar, G.R., Allam, A.R., Muraleedharan, M.V., Jaya Kumar, R.V. \& Venkat, Y. (2009) "Electronic medical records and hospital management systems for management of diabetes", Diabetes \& Metabolic Syndrome: Clinical Research \& Reviews, Vol.3 Iss 1 pp 55 - 59.

Thielst, C. B. (2007a). The future of healthcare technology. Journal of Healthcare Management, 52(1), 7-9.

Thielst, C. B. (2007b). The new frontier of electronic, personal, and virtual health records. Journal of Healthcare Management, 52(2), 75-78.

\section{ACKNOWLEDGMENT}

The authors would like to thank the relevant parties that have directly or indirectly contributed to the success of this paper. Besides that, the authors wish to express their gratitude to the anonymous referees for their helpful comments and numerous suggestions to improve the paper.

\section{Authors Biography}

Nik Azliza Nik Ariffin is a senior lecturer in Records Management and Information System at the Faculty of Information Management at University Technology MARA (UiTM). She is currently pursuing her study in PhD and mainly specializes in Electronic Medical Records Management System. She is a graduate of the International Islamic University Malaysia and University Technology MARA (UiTM). Her research interests are in the areas of electronic records management, health information management, records management and medical records management. 